\title{
Nuclear translocation of annexin 1 following oxygen-glucose deprivation-reperfusion induces apoptosis by regulating Bid expression via p53 binding
}

\author{
Xing Li ${ }^{1,2,3,4}$, Yin Zhao ${ }^{1,4}$, Qian Xia ${ }^{1,2,3}$, Lu Zheng ${ }^{1,2,3}$, Lu Liu ${ }^{1,2,3}$, Baoming Zhao ${ }^{1,2,3}$ and Jing Shi*,1,2,3
}

Previous data have suggested that the nuclear translocation of annexin 1 (ANXA1) is involved in neuronal apoptosis after ischemic stroke. As the mechanism and function of ANXA1 nuclear migration remain unclear, it is important to clarify how ANXA1 performs its role as an apoptosis 'regulator' in the nucleus. Here we report that importazole (IPZ), an importin $\beta$ (Imp $\beta$ )-specific inhibitor, decreased ANXA1 nuclear accumulation and reduced the rate of neuronal death induced by nuclear ANXA1 migration after oxygen-glucose deprivation-reoxygenation (OGD/R). Notably, ANXA1 interacted with the Bid (BH3-interacting-domain death agonist) promoter directly; however; this interaction could be partially blocked by the p53 inhibitor pifithrin- $\alpha$ (PFT- $\alpha$ ). Accordingly, ANXA1 was shown to interact with p53 in the nucleus and this interaction was enhanced following OGD/R. A luciferase reporter assay revealed that ANXA1 was involved in the regulation of $\mathrm{p53}$-mediated transcriptional activation after OGD/R. Consistent with this finding, the nuclear translocation of ANXA1 after OGD/R upregulated the expression of Bid, which was impeded by IPZ, ANXA1 shRNA, or PFT- $\alpha$. Finally, cell-survival testing demonstrated that silencing ANXA1 could improve the rate of cell survival and decrease the expression of both cleaved caspase-3 and cleaved poly(ADP-ribose) polymerase. These data suggested that Imp $\beta$ dependent nuclear ANXA1 migration participates in the OGD/R-dependent induction of neuronal apoptosis. ANXA1 interacts with p53 and promotes p53 transcriptional activity, which in turn regulates Bid expression. Silencing ANXA1 decreases the expression of Bid and suppresses caspase-3 pathway activation, thus improving cell survival after OGD/R. This study provides a novel mechanism whereby ANXA1 regulates apoptosis, suggesting the potential for a previously unidentified treatment strategy in minimizing apoptosis after OGD/R.

Cell Death and Disease (2016) 7, e2356; doi:10.1038/cddis.2016.259; published online 1 September 2016

Ischemia-reperfusion is a well-recognized pathological condition that is characterized by an initial deprivation of blood supply to an area or organ followed by subsequent vascular restoration and concomitant reoxygenation of downstream tissues. ${ }^{1,2}$ Ischemia-reperfusion occurs during various complications of vascular diseases, such as stroke and myocardial infarction. ${ }^{1,3-5}$ Oxygen-glucose deprivation-reoxygenation (OGD/R) is an accepted model for studying ischemia-reperfusion in vitro. ${ }^{6-8}$ Previous findings have indicated that annexin 1 (ANXA1) is involved in neuronal apoptosis after $\mathrm{OGD} / \mathrm{R}$, but the underlying mechanism remains unclear. $^{9}$ Therefore, it is important to clarity the regulatory role(s) of ANXA1 in OGD/R-mediated apoptosis.

As a $\mathrm{Ca}^{2+}$ - and phospholipid-binding protein, ${ }^{10,11}$ ANXA1 performs different roles depending on its subcellular localization. Kim et al. ${ }^{12}$ reported that phorbol 12-myristate 13-acetate (PMA)-induced ANXA1 nuclear translocation might participate in the regulation of cell proliferation and differentiation.
Wang et al. ${ }^{13}$ revealed that kirenol and prednisolone promote the nuclear localization of ANXA1, which then interacts with $N F-K B$ to inhibit NF-KB activity, reducing cytokine expression and thereby attenuating the inflammation of collagen-induced arthritis. Results from our previous study indicated that ANXA1 migration into the nucleus and its regulation of Bid (BH3-interacting-domain death agonist) gene expression were involved in apoptosis. ${ }^{9}$ However, exactly how ANXA1 regulates Bid expression and induces apoptosis after OGD/R remains unclear.

Bid can participate in neuronal apoptosis induced by brain ischemia, ${ }^{14,15}$ and its expression is regulated by the transcription factor p53. ${ }^{16,17}$ The genomic loci for both the human and the mouse Bid genes contain p53-binding, DNA-response elements that bind p53 and mediate p53-dependent transactivation of reporter genes. ${ }^{18,19}$ Unlike all other known BH3-only proteins, Bid needs to be cleaved by caspase- 8 or other proteases into tBid to become activated, whereupon it

\footnotetext{
${ }^{1}$ Department of Neurobiology, Tongji Medical College, Huazhong University of Science and Technology, Wuhan, People's Republic of China; ${ }^{2}$ Key Laboratory of Neurological Diseases, Ministry of Education, Wuhan, People's Republic of China and ${ }^{3}$ Institute for Brain Research, Huazhong University of Science and Technology, Wuhan, People's Republic of China

*Corresponding author: J Shi, Department of Neurobiology and Key Laboratory of Neurological Disease of Ministry of Education, Tongji Medical College, Huazhong University of Science and Technology, 13 Hangkong Road, Wuhan 430030, People's Republic of China. Tel: +86 27 83650545; Fax: +86 27 83692608;

E-mail: sj@mails.tjmu.edu.cn

${ }^{4}$ These authors contributed equally to this work.

Abbreviations: ANXA1, annexin 1; OGD/R, oxygen-glucose deprivation-reoxygenation; Co-IP, co-immunoprecipitation; ChIP, chromatin immunoprecipitation assay; IPZ, importazole; IMT, ivermectin; shRNA, short hairpin RNA; Scr, scrambled control DNA; PARP, poly(ADP-ribose) polymerase; PFT- $\alpha$, pifithrin- $\alpha$; PMA, phorbol 12-myristate 13-acetate; $\operatorname{Imp} \beta$, importin $\beta$; PVDF, polyvinylidene difluoride

Received 01.2.16; revised 18.7.16; accepted 29.7.16; Edited by A Verkhratsky
} 
translocates to the mitochondrial outer membrane ${ }^{20}$ where it leads to the activation of Bax/Bak, thereby inducing the cytosolic release of cytochrome $c$ from the mitochondria after ischemia. $^{21,22}$ In turn, the released cytochrome $c$ ultimately leads to the cleavage and activation of caspase- 9 and caspase $-3,{ }^{23}$ representing the final steps of the intrinsic caspase pathway that has been previously shown to regulate ischemic cell death. ${ }^{15,24}$

The p53 tumor suppressor protein acts as a major defense mechanism against cancer. ${ }^{25}$ Among its most distinctive features is its ability to elicit both apoptotic death and cell cycle arrest. $^{26}$ Numerous p53 co-factors have been implicated in cellular processes, and the activation or suppression of these co-factors determines the fate of a cell. ${ }^{27,28}$ Whether ANXA1 represents one of the p53 co-factors involved in the regulation of Bid expression and how ANXA1 might interact with p53 to coregulate Bid expression have yet to be determined.

In this study, we investigated the role of ANXA1 in neuronal apoptosis after OGD/R. Specifically, we examined the requirement for importin $\beta(\operatorname{Imp} \beta)$-activation in nuclear migration of ANXA1 and determined the interaction and coregulatory status of ANXA1 and p53 in the cell nucleus with respect to p53 transcriptional activity, Bid expression, and eventual caspase-3/poly(ADP-ribose) polymerase (PARP) activation after OGD/R.

\section{Results}

Nuclear ANXA1 translocation mediated by functional Imp $\boldsymbol{\beta}$ induces neuronal death after OGD/R. Impa and $-\beta$ are considered classic nuclear envelope transporters. We used the specific $\operatorname{Imp} \beta$ inhibitor importazole (IPZ) ${ }^{29}(8 \mu \mathrm{M})$ and the specific Impa inhibitor ivermectin (IMT) ${ }^{30}(25 \mu \mathrm{M})$ to separately block the functions of $\operatorname{Imp} \beta$ and Impa. To investigate the subcellular location of ANXA1 after OGD/R, we extracted cytoplasmic and nuclear proteins from primary cultured neurons. As shown in Figures $1 \mathrm{a}$ and b, OGD/R increased the expression of ANXA1 in whole-cell lysates. Furthermore, OGD/R increased ANXA1 nuclear translocation without effect on its expression in the cytoplasm. Treatment with IPZ, but not IMT, reduced ANXA1 expression in the nucleus. Immunofluorescence analysis also indicated that under normal conditions, ANXA1 primarily localized to the cytoplasm in neuronal cells and IPZ could inhibit OGD/Rinduced nuclear ANXA1 translocation (Figure 1c). To determine the effect of ANXA1 on neuron death after $\mathrm{OGD} / \mathrm{R}$, the adenovirus vector carrying rat $A N X A 1$ gene was constructed and overexpression of ANXA1 in primary cultured neurons was confirmed by western blot (Supplementary Figures S1a-c). Then, we performed propidium iodide $(\mathrm{PI})$ staining followed by $A N X A 1$ transfection, $I P Z$, or (and) OGD/R treatment. The results revealed that OGD/R-induced cell death and that overexpression of ANXA1 associated with OGD/R promoted neuron death. In contrast, treatment with IPZ markedly decreased nuclear ANXA1 translocation and subsequent cell death after OGD/R in neuron (Figures 1d and e). Taken together, these results suggested that nuclear ANXA1 migration depended upon functional $\operatorname{Imp} \beta$ and was involved in OGD/R-induced neuronal cells death.

ANXA1 accumulates on the Bid gene promoter. To determine the function of ANXA1 in the nucleus, and especially potential physical interactions with apoptosisrelated gene loci, we performed chromatin immunoprecipitation (ChIP) assays to isolate ANXA1-DNA-binding complexes from primary cultured rat neurons, after which ChIP-Seq was performed on a Solexa Genome Analyzer (Solexa, Cambridge, UK) to identify the complex components in cooperation with BGI-Shenzhen (China). We identified 622 different genes that interacted with ANXA1 under normal physiological conditions and 1284 genes under OGD/R conditions, of which 251 genes were overlapping (Figure 2a). Gene ontology (GO) analyses were also used to explore the functional annotations of these target genes. These genes were classified into six categories, based on their involvement in different biological processes (Figure $2 b$ ). We found that ANXA1 potentially interacts with 22 different apoptosisrelated genes and 36 cell death-related genes under normal conditions, and with 47 apoptosis-related genes and 76 cell death-related genes following OGD/R (Figure 2b). Comparison of the ChIP-Seq data with the UCSC Genome Browser for the rat genome (March 2012; RGSC 5.0/rn5) revealed that ANXA1 located to the Bid gene promoter domain following OGD/R (Figure 2c). To confirm these results, a ChIP-polymerase chain reaction (PCR) assay was performed. As shown in Figures $2 d$ and e, ANXA1 directly associated with the Bid gene promoter under both normoxic and OGD/R conditions in human embryonic kidney 293 (HEK293) cells, although the total level of ANXA1 binding to the Bid promoter was increased after OGD/R.

ANXA1 promotes the transcription and translation of Bid under OGD/R conditions. To examine the function of ANXA1 on Bid expression, we treated primary cultured neurons and HEK293 cells with IPZ to decrease nuclear ANXA1 translocation and then examined Bid expression. As shown in Figure $3 a$, quantitative real-time PCR (qPCR) demonstrated that the upregulation of ANXA1 after OGD/R increased Bid expression, but treatment with IPZ downregulated the expression of $B i d$. Similar trends were observed for protein levels (Figures $3 b$ and $c$ ). To block the expression of ANXA1 in primary cultured neurons, we constructed an adenoviral vector that encodes short hairpin RNA (shRNA) against rat $A N X A 1$. Western blot analysis showed that treatment with shRNA resulted in observable knockdown (Supplementary Figures S2a and b). Then, we examined the expression of Bid. Downregulation of ANXA1 resulted in markedly decreased of Bid expression in primary cultured neurons under OGD/R conditions (Figures $3 d-f$ ). In addition, we used human $A N X A 1$ shRNAs to knock down ANXA1 expression in HEK293 cells. As shown in Supplementary Figures $\mathrm{S} 3 \mathrm{a}$ and $\mathrm{b}$, the no. 2 shRNA against $A N X A 1$ efficiently decreased ANXA1 expression. Then, we examined the expression of Bid. Silencing $A N X A 1$ with shRNA no. 2 decreased Bid expression under OGD/R conditions at both the mRNA and protein level in HEK293 cells (Figures 3d-f). 
a

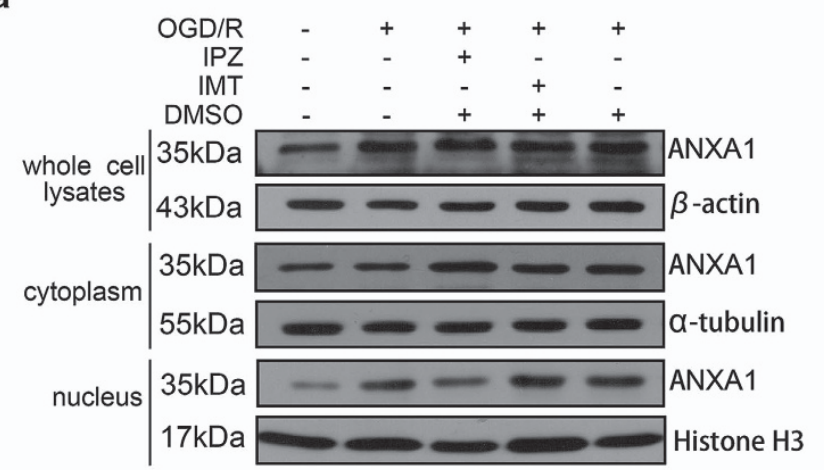

b

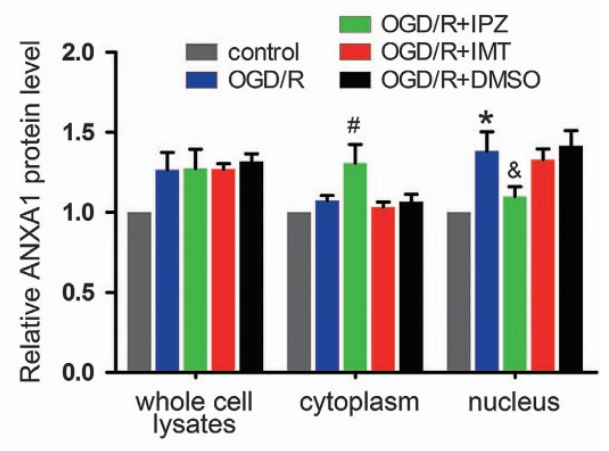

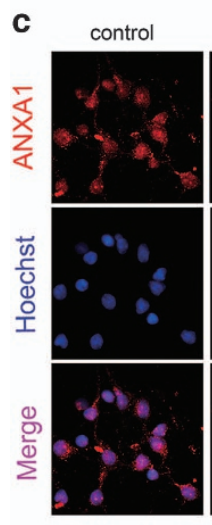

OGD/R

IPZ+OGD/R
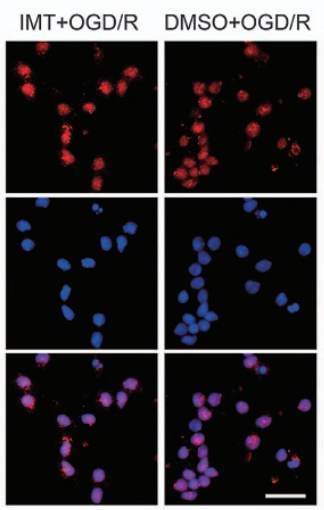

e
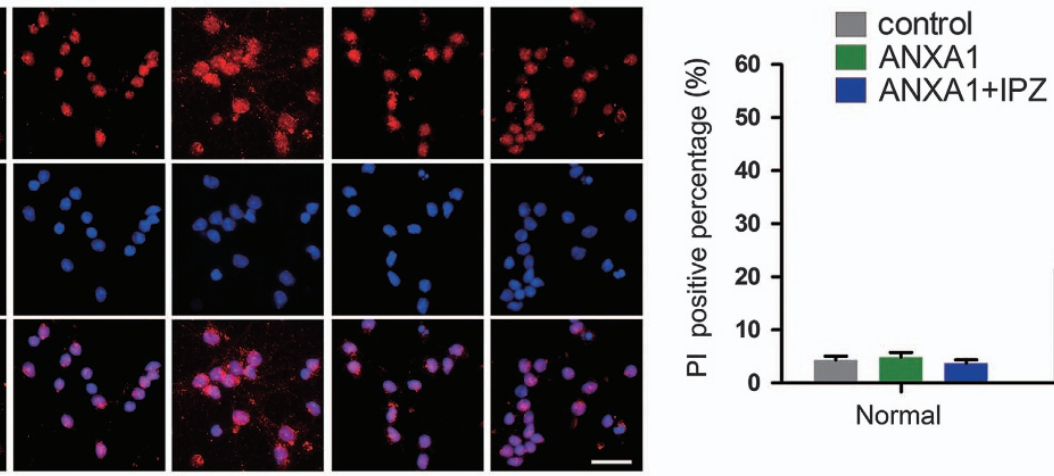

$\overbrace{}^{\circ}{ }^{60}-\square$ ANXA1+IPZ
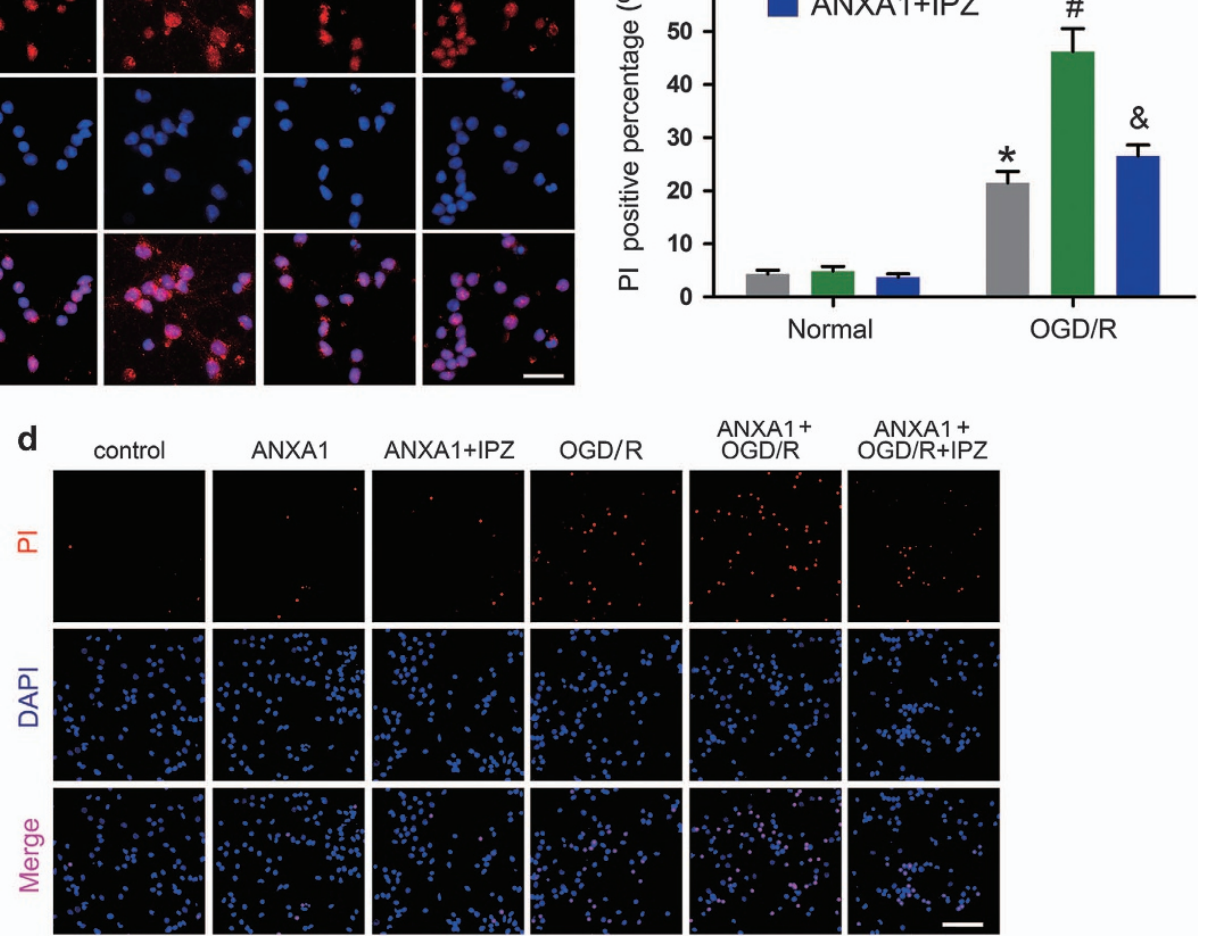

ANXA1 +
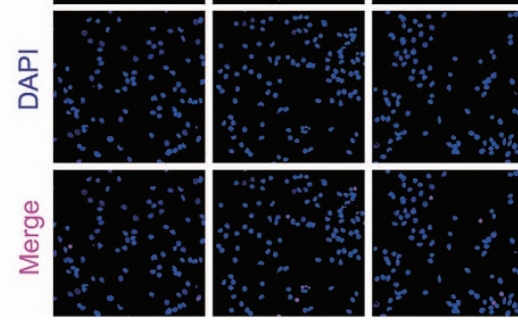

Figure 1 Expression of ANXA1 and neuronal death after OGD/R. (a) Western blots showing ANXA1 expression in primary cultured neurons treated with IPZ or IMT after $\mathrm{OGD} / \mathrm{R}$. (b) Statistical analysis of the data shown in (a). The data shown are expressed as the means \pm S.E.M. from three independent experiments. ${ }^{*} P<0.05$ versus control, ${ }^{\#} P<0.05$ versus $\mathrm{OGD} / \mathrm{R}$ and ${ }^{\&} P<0.05$ versus $\mathrm{OGD} / \mathrm{R}$. (c) Representative images of ANXA1 expression in primary cultured neurons treated with IPZ or IMT after OGD/R. Scale bar $=25 \mu \mathrm{m}$. (d and e) Representative PI staining and statistical analysis show the effect of ANXA1 overexpression on cell death in primary cultured neurons after OGD/R. Cell death was visualized by PI staining. Scale bar $=50 \mu \mathrm{m}$. The data are expressed as the means \pm S.E.M. from three independent experiments. ${ }^{*} P<0.05$ versus control, ${ }^{\#} P<0.05$ versus $\mathrm{OGD} / \mathrm{R}$ and ${ }^{\circledR} P<0.05$ versus ANXA1+OGD/R

ANXA1 interacts with p53 in the nucleus following OGD/R. The p53 protein has been reported to function as a Bid gene transcription factor. To examine the role of $p 53$, we first determined the subcellular location of p53 in primary cultured neurons following OGD/R. We found that OGD/R increased the expression of p53 in whole-cell lysates and promoted its nuclear accumulation (Figures $4 a$ and b). We then constructed the plasmids ANXA1-His and p53-GFP (green fluorescent protein) to assess the relationship between ANXA1 and p53 in HEK293 cells. Coimmunoprecipitation (Co-IP) results revealed that ANXA1 associated with p53 (Figure 4c). In addition, we examined the subcellular location of the interaction in primary cultured neurons, which suggested that OGD/R increased ANXA1p53 interactions and that this interaction occurred primarily in the nucleus (Figure 4d). Immunofluorescence analysis also suggested that in primary cultured neurons, endogenous ANXA1 colocalized with p53 in the nucleus after OGD/R (Figures $4 \mathrm{e}$ and $\mathrm{f}$ ). In addition, we transfected the plasmids pjRed-ANXA1 (red) and pEGFP-p53 (green) into HEK293 cells to confirm the interaction by immunofluorescence. These results also indicated that OGD/R increased the 


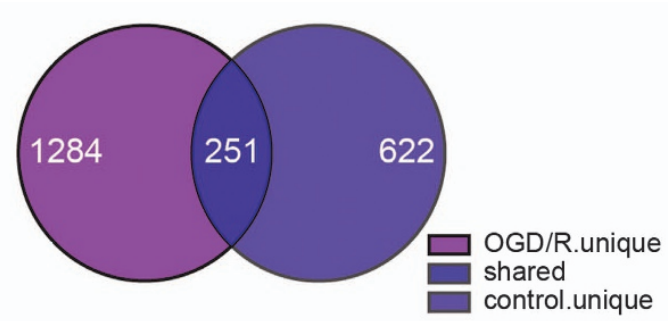

b

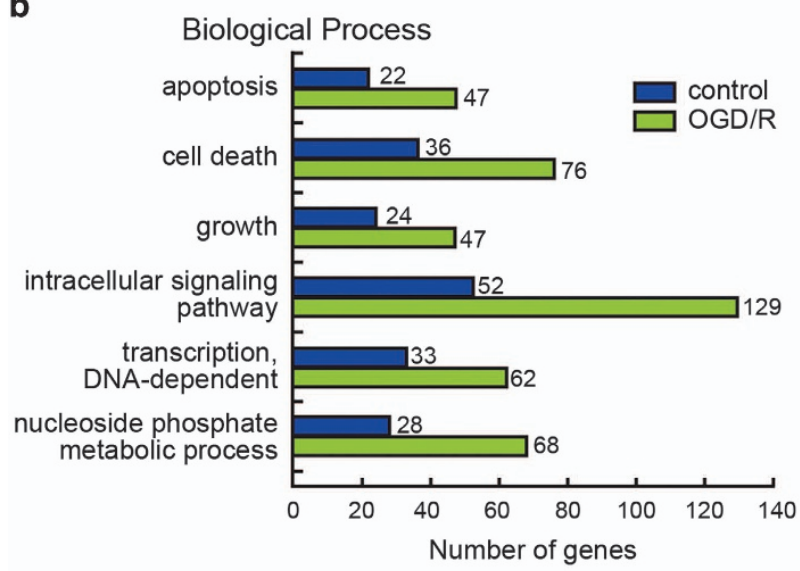

C

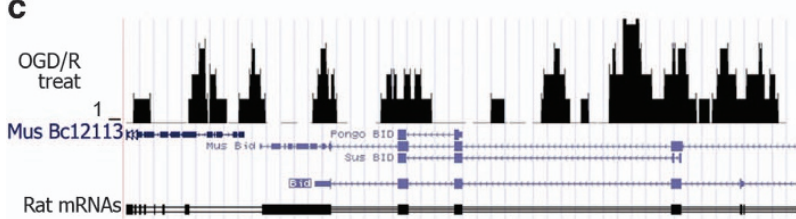

d

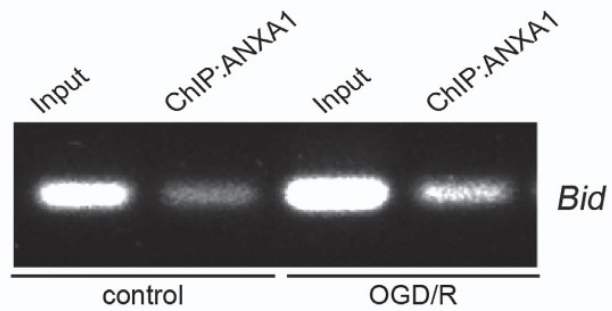

e

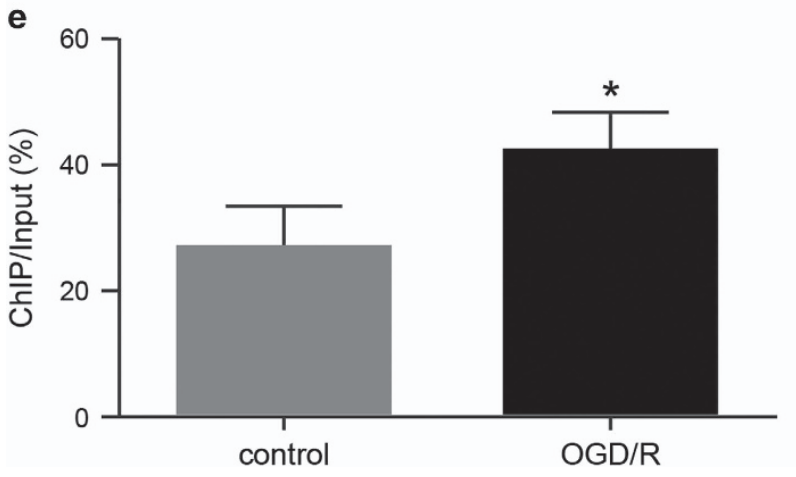

interaction between ANXA1 and p53 in the nucleus (Figures $4 \mathrm{~g}$ and $\mathrm{h}$ ).

ANXA1 regulates p53 transcriptional activation after OGD/R. To examine the function of the ANXA1-p53
Figure 2 Effect of OGD/R on ANXA1 accumulation on the Bid gene promoter. (a) Venn diagram of the overlap among all ANXA1-bound genes identified by ChIPSeq in primary cultured neurons treated without or with OGD/R. The genes that bound ANXA1 from the normal and OGD/R group ( $n=3$ independent samples) are shown. (b) GO enrichment of ANXA1-bound proteins compared with the total genes from the rat genome. Significant enrichments of $\mathrm{GO}$ terms were obtained for the biological processes shown. (c) UCSC Genome Browser tracks depicting ANXA1 ChIP-Seq peaks at representative Bid gene loci under OGD/R conditions. (d) ChIPPCR results show the interaction between ANXA1 and the Bid gene promoter after OGD/R in HEK293 cells. (e) Statistical analysis of the data shown in (d). The data are expressed as the means \pm S.E.M. from three independent experiments. ${ }^{*} P<0.05$ versus control

interaction, we treated HEK293 cells with pifithrin- $a$ (PFT- $a$ ) $(20 \mu \mathrm{M})$ to inhibit the function of p53. ChIP-PCR was used to assess the interaction between ANXA1 and the Bid gene promoter. The results revealed that the interaction between ANXA1 and the Bid promoter was inhibited by PFT- $a$ (Figures $5 \mathrm{a}$ and $\mathrm{b}$ ). A luciferase reporter assay was then conducted to examine p53 transcriptional activity in HEK293 cells, which suggested that compared with normal conditions, OGD/R increased p53 transcriptional activation, and that compared with OGD/R alone, ANXA1 overexpression+ OGD/R significantly increased p53 transcriptional activation $(P<0.05$; Figure $5 \mathrm{c})$. In contrast, shRNA-mediated ANXA1 silencing demonstrated that compared with OGD/R +scrambled control DNA (Scr), OGD/R+ANXA1 shRNA decreased p53 transcriptional activation (Figure 5d). Taken together, these results suggested that ANXA1 regulated p53 transcriptional activation and that the binding between ANXA1 and the Bid gene promoter could be blocked by p53 inhibition.

ANXA1 coregulates Bid with p53. Despite demonstrating their association and DNA binding, the function of the ANXA1-p53 interaction remained unclear. To address this issue, we examined the expression of Bid using qPCR and western blot analysis. As shown in Figure $6 a$, OGD/R significantly increased Bid expression, whereas PFT- $a$ markedly decreased the expression of Bid mRNA. Similar results were observed by western blot in neuron analysis (Figures $6 \mathrm{~b}$ and $\mathrm{c}$ ). These results were also confirmed in HEK293 cells (Figures 6a-c). In addition, we found that the upregulation of Bid induced by ANXA1 overexpression was reversed by PFT- $a$ under OGD/R conditions (Figures $6 d-f$ ). Finally, we demonstrated that along with ANXA1 downregulated by used $A N X A 1$ shRNA, the level of Bid expression was also decreased after OGD/R. Treatment with $A N X A 1$ shRNA+PFT- $a$ showed that the level of Bid expression was lower compared with when treated with ANXA1 shRNA after OGD/R in primary cultured neurons and HEK293 cells (Figures 6g-i).

ANXA1 regulates cell apoptosis via the caspase-3 pathway. Previous data have indicated that nuclear ANXA1 migration upregulates Bid expression, which is involved in OGD/R-induced cell apoptosis. However, the exact apoptosis pathway involved in this process had not been determined. The caspase-3-PARP is a classic apoptosis pathway. We 

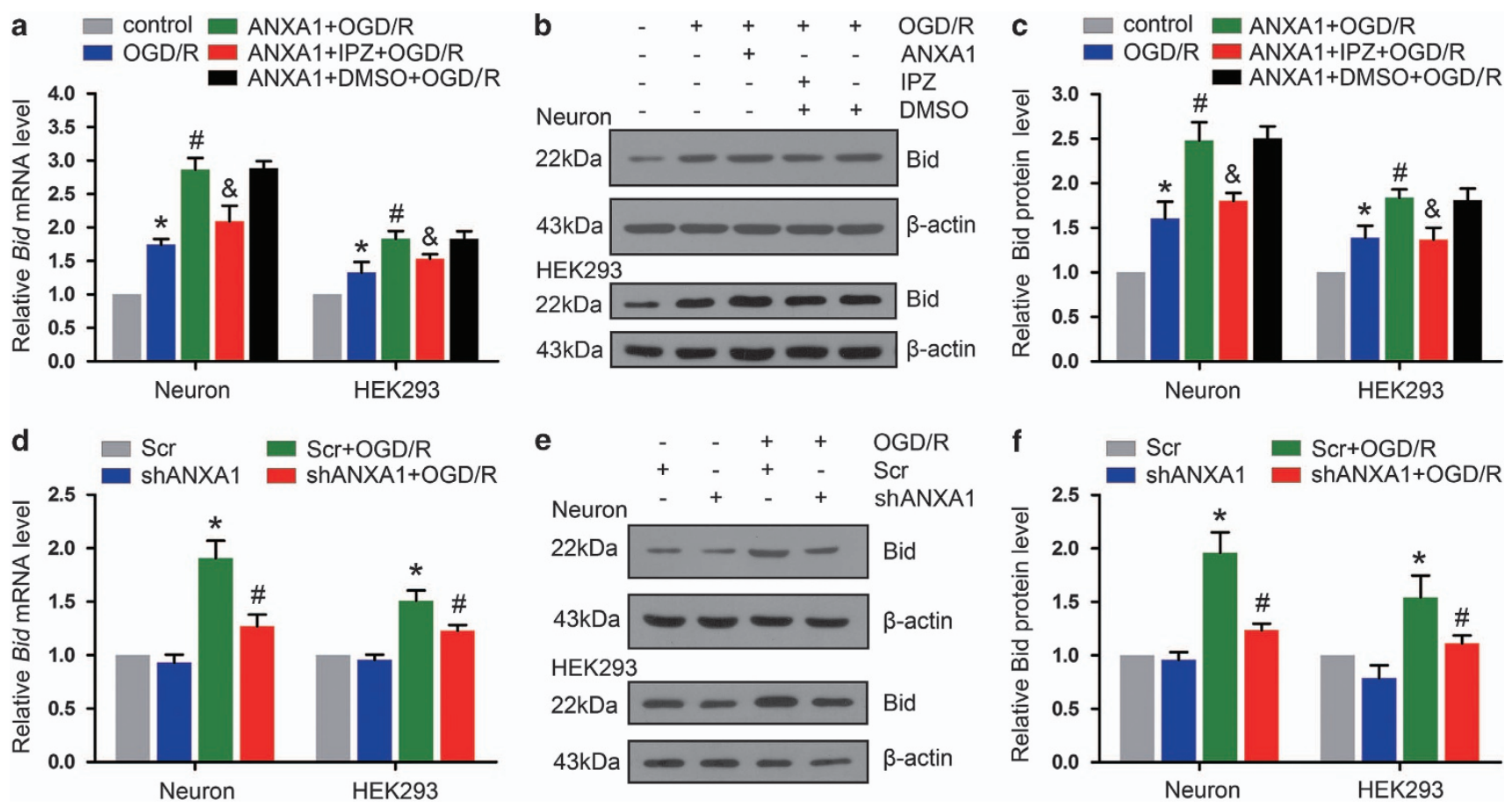

Figure 3 Effect of ANXA1 nuclear migration on Bid expression. (a) qPCR shows the effects of ANXA1 overexpression and IPZ treatment on Bid mRNA expression after $\mathrm{OGD} / \mathrm{R}$. The data are expressed as the means \pm S.E.M. from three independent experiments. ${ }^{*} P<0.05$ versus control, ${ }^{\sharp} P<0.05$ versus $0 \mathrm{GD} / \mathrm{R}$ and ${ }^{\circledR} P<0.05$ versus ANXA1 $+O G D / R$. (b) Western blot results showing the effects of ANXA1 overexpression and IPZ on Bid protein expression after OGD/R. (c) Statistical analysis of the data shown in (b). The data are expressed as the means \pm S.E.M. from three independent experiments. ${ }^{*} P<0.05$ versus control, ${ }^{\#} P<0.05$ versus 0 GD/R and ${ }^{\&} P<0.05$ versus ANXA1+OGD/R. (d) qPCR results showing the expression of Bid mRNA after treatment with ANXA1 shRNA. The data are expressed as the means \pm S.E.M. from three independent experiments. ${ }^{*} P<0.05$ versus $S c r$ and ${ }^{\#} P<0.05$ versus Scr+OGD/R. (e) Western blot showing expression of the Bid protein after treatment with ANXA1 shRNA. (f) Statistical analysis of the data shown in (e). The data are expressed as the means \pm S.E.M. from three independent experiments. ${ }^{*} P<0.05$ versus Scr and ${ }^{\#} P<0.05$ versus Scr+OGD/R

found that ANXA1 overexpression increased the expression of cleaved caspase-3 and PARP in neuron and that this increase was reduced by ANXA1 shRNA following OGD/R (Figure 7a). In addition, ANXA1-induced caspase-3 activation was downregulated by treatment with PFT- $a(20 \mu \mathrm{M})$ or the Bid inhibitor Bl-6C9 $(2 \mu \mathrm{M})$ in primary cultured neurons (Figure 7b). These results were also confirmed in HEK293 cells (Figures $7 a$ and $b$ ). Taken together, these data indicated that ANXA1 regulated caspase-3 activation via the p53-Bid pathway under OGD/R conditions. Similar trends were observed by $\mathrm{PI}$ staining, and the results indicated that ANXA1-induced cell apoptosis was impeded by treatment with $\mathrm{PFT}-a, \mathrm{BI}-6 \mathrm{C} 9$, or ANXA1 shRNA in primary cultured neurons (Figures $7 c$ and $d$ ). These results were also confirmed in HEK293 cells by flow cytometry (Figures $7 e$ and f).

\section{Discussion}

In the present study, we elucidated the role of nuclear ANXA1 migration in OGD/R-induced cell apoptosis. Specifically, we found that following OGD/R, ANXA1 translocated from the cytoplasm to the nucleus and that this migration could be blocked by the $\operatorname{Imp} \beta$ inhibitor IPZ. In the nucleus, ANXA1 interacted with the transcription factor p53. Conditions of OGD/R+ANXA1 increased the transcriptional activity of p53, thereby increasing Bid expression. This process was inhibited by the p53 inhibitor, PFT- $a$. Notably, we found that silencing ANXA1 with shRNA could also decrease Bid expression and enhance cell survival after OGD/R.

The first part of this study was aimed to address the mechanism by which ANXA1 influences OGD/R-induced cell apoptosis. We found that OGD/R not only increased ANXA1 expression in whole-cell lysates but also increased the accumulation of ANXA1 in the nucleus. Previous data indicated that the nuclear translocation of ANXA1 was due to stimulation by the mitogen PMA. PMA induced the expression and phosphorylation of ANXA1, which then increased ANXA1 nuclear migration. ${ }^{12}$ As OGD/R stimulation activates TRPM7 function and ANXA1 has been shown to be phosphorylated by the TRPM7 kinase, ${ }^{31,32}$ we speculated that ANXA1 upregulation was due to TRPM7 kinase activation following OGD/R. Consistent with this possibility, our previous data revealed that truncation of the TRPM7 kinase decreased nuclear ANXA1 translocation. ${ }^{9}$ In addition, we demonstrated that the decreased cell-survival rate observed after OGD/R was due to nuclear ANXA1 migration.

The full-length ANXA1 consists of 346 amino acids and contains an N-terminal domain and a C-terminal core domain. The central domain of ANXA1 is composed of four repeat sequences ${ }^{33,34}$ Previous studies have provided evidence that the ANXA1-dependent inhibition of local and systemic inflammatory processes could be recapitulated using the ANXA1 mimetic peptide Ac 2-26, which comprises the first 25 
a

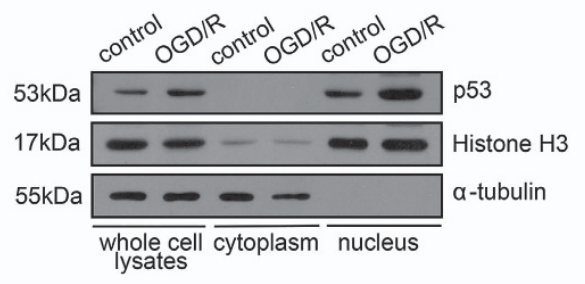

d

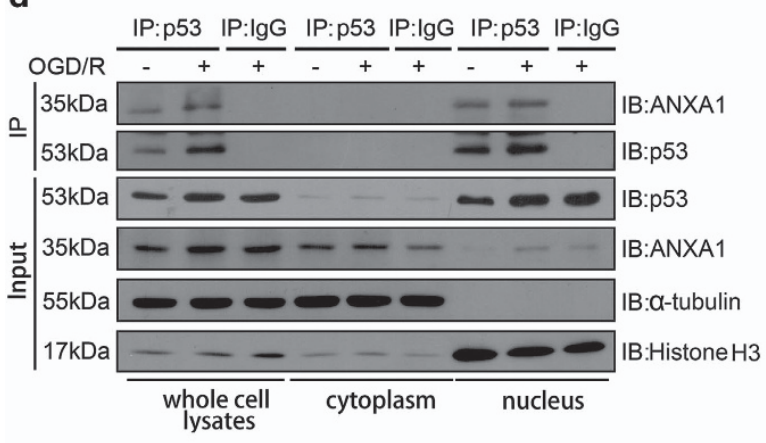

e

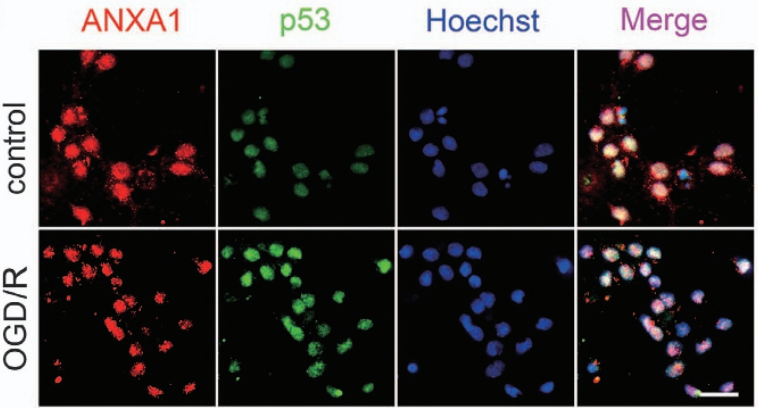

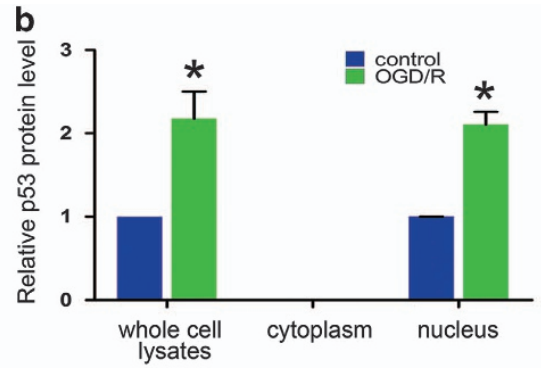

C

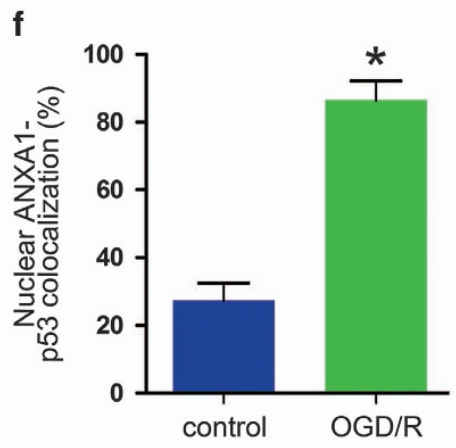

h
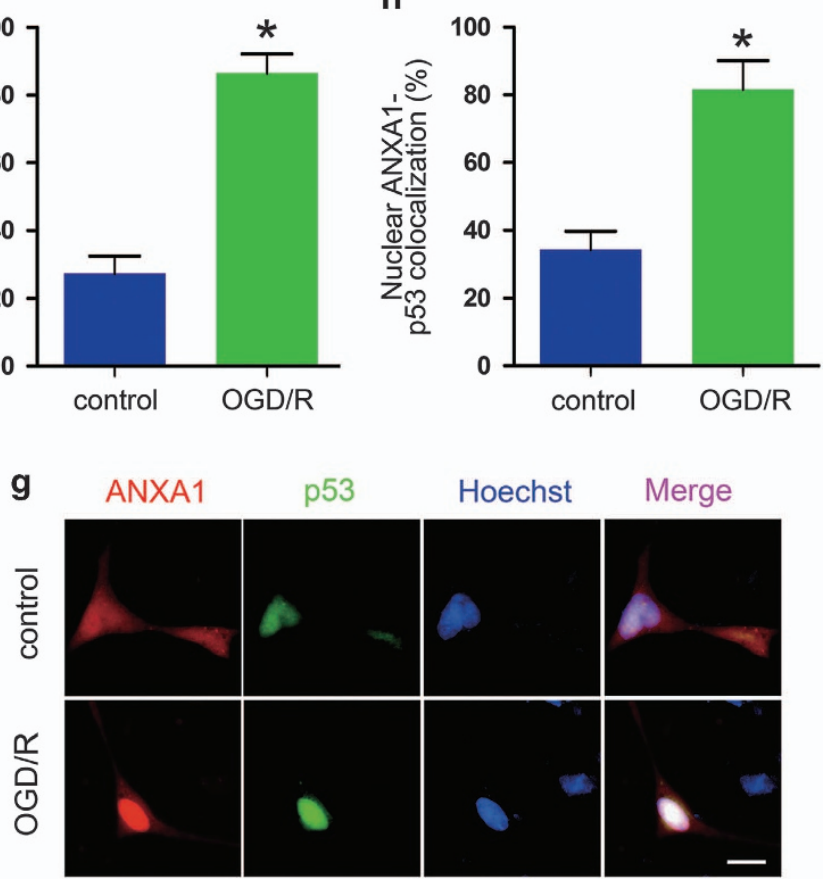

Figure 4 ANXA1 interacts with p53 in the nucleus after OGD/R. (a) Western blot results demonstrating the expression of p53 in the cytoplasm and nucleus in primary cultured neurons treated without or with OGD/R. (b) Quantitative densitometry analysis of the p53 expression shown in (a). The data are expressed as the means \pm S.E.M. from three independent experiments. ${ }^{*} P<0.05$ versus control. (c) Representative Co-IP results show the interactions of ANXA1 with p53 in HEK293 cells transfected with pcDNA-ANXA1 (His-tagged) and pEGFP-p53 (GFP-tagged) treated without or with OGD/R. (d) Representative Co-IP results show the interactions of ANXA1 with p53 in primary cultured neurons treated without or with OGD/R. (e) Immunofluorescence shows the colocalization of endogenous p53 and ANXA1 in primary cultured neurons treated with or without $\mathrm{OGD} / \mathrm{R}$. Scale bar $=50 \mu \mathrm{m}$. (f) The nuclear colocalization of endogenous ANXA1 and p53 was quantitated as a graph. The data are expressed as the means \pm S.E.M. from three independent experiments. ${ }^{*} P<0.05$ versus control. (g) Immunofluorescence shows the colocalization of ANXA1 and p53 in HEK293 cells transfected with pjRed-ANXA1 and pEGFP-p53 treated with or without OGD/R. Scale bar $=50 \mu \mathrm{m}$. (h) The nuclear colocalization of endogenous ANXA1 and p53 was quantitated as a graph. The data are expressed as the means \pm S.E.M. from three independent experiments. ${ }^{*} P<0.05$ versus control

amino acids of ANXA1. ${ }^{35-37}$ In this study, we found that the nuclear translocation of overexpressed ANXA1 might increase neuronal cells death after OGD/R, and we speculate that ANXA1-induced cell death was due to its remaining amino-acid sequence. Considering that ANXA1 lacks a classical nuclear localization signal, we further speculate that nuclear ANXA1 translocation was due to a non-classical translocation pathway. In this study, we also found that the classic nuclear-migration pathway inhibitor IMT had no effect on ANXA1 nuclear translocation, but that the non-classicalmigration pathway inhibitor IPZ, which blocked the function of $\operatorname{Imp} \beta$, decreased nuclear ANXA1 accumulation. Identification of the specific amino-acid sequences that perform key roles in regulating nuclear ANXA1 translocation and cell death induction remain to be determined in future studies.
We next investigated ANXA1 function in the nucleus using ChIP-Seq analysis. We immunoprecipitated ANXA1-DNA complexes with an ANXA1 antibody and then determined the bound DNA components using high-throughput sequencing. GO analysis indicated that ANXA1 might be involved in various cellular processes, including cell apoptosis. Considering that ANXA1 does not contain a DNA-binding element, we speculate that ANXA1 might associate with transcription factors to regulate gene expression. Consistent with this hypothesis, we found that ANXA1 interacted with p53, which is well known as a Bid gene transcription factor, ${ }^{16}$ and our findings demonstrated that ANXA1 increased p53-dependent transcriptional activation. These data indicated that ANXA1 interacted with p53 and enhanced transcriptional activation and that this complex regulated Bid expression. 
a

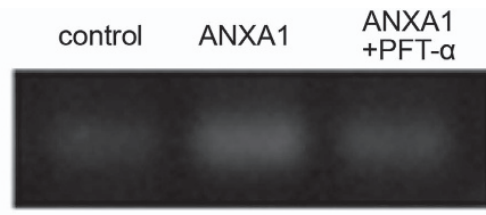

ChIP:ANXA1

Bid primers

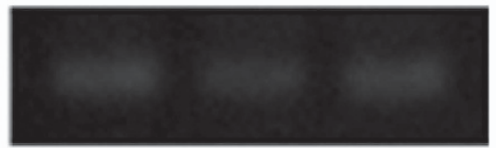

Input

b

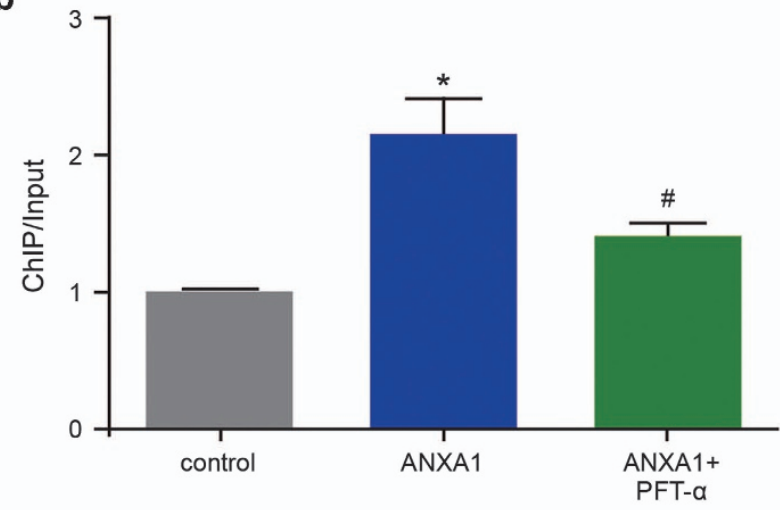

C

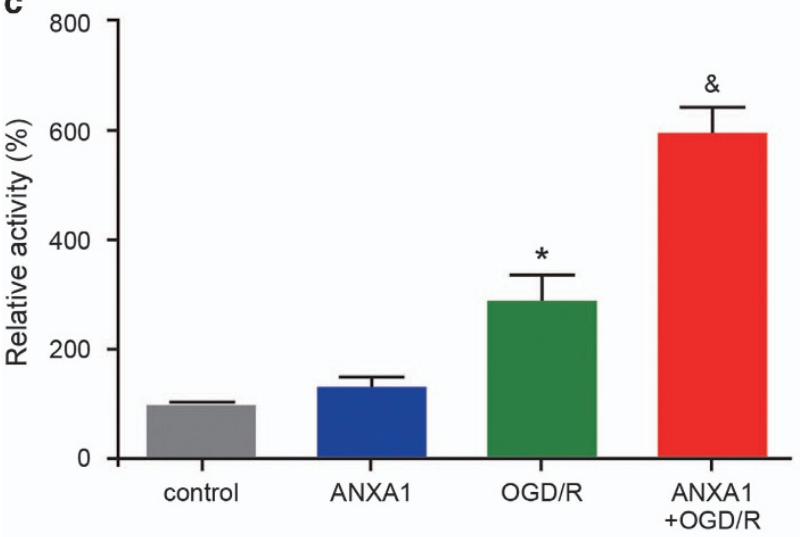

d

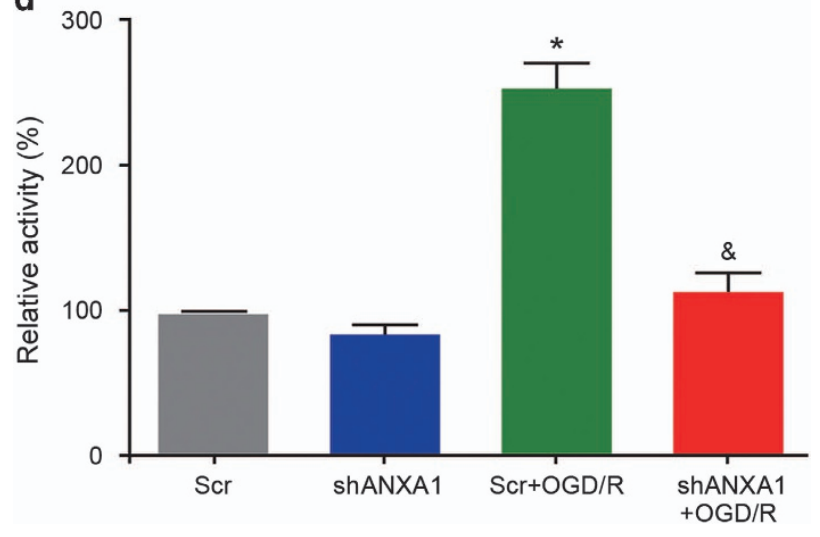

Figure 5 Effect of ANXA1 on p53 transcriptional activity. (a) ChIP-PCR analysis showing the combination of ANXA1 with Bid following treatment of HEK293 cells with ANXA1 overexpression and PFT- $\alpha$. (b) Statistical analysis of the data shown in (a). The data are expressed as the means \pm S.E.M. from three independent experiments. ${ }^{*} P<0.05$ versus control and ${ }^{\#} P<0.05$ versus ANXA1. (c) The luciferase assays show the transcriptional activity of $p 53$ following treatment with ANXA1 after OGD/R in HEK293 cells. The data are expressed as the means \pm S.E.M. from three independent experiments. ${ }^{*} P<0.05$ versus control and ${ }^{\#} P<0.05$ versus 0 GD/R. (d) The luciferase assays show the transcriptional activity of p53 following treatment with ANXA1 shRNA in HEK293 cells. The data are expressed as the means \pm S.E.M. from three independent experiments. ${ }^{*} P<0.05$ versus $S c r$ and ${ }^{\&} P<0.05$ versus $\mathrm{Scr}+\mathrm{OGD} / \mathrm{R}$

Taken together, these data suggested that the non-classical mechanism by which ANXA1 is translocated into the nucleus involves transport by $\operatorname{Imp} \beta$ function. In addition, the results contribute to our knowledge of ANXA1 by elucidating the function of its nuclear migration after OGD/R. This study is the first to demonstrate that ANXA1 interacts with p53 to coregulate Bid expression and induce cell death after OGD/ $\mathrm{R}$ via the caspase-3 pathway (Figure 8 ). Collectively, these observations provide a more comprehensive understanding of ANXA1 function in cell apoptosis after OGD/R and strongly suggest that decreasing nuclear ANXA1 migration might be a useful therapeutic approach for treating ischemic stroke.

\section{Materials and Methods}

Reagents and antibodies. IPZ was obtained from Calbiochem (Darmstadt, Germany; 401105). IMT, PFT- $\alpha$, and BI-6C9 were obtained from Sigma-Aldrich China (Shanghai, China; 18898, P4236, and B0186, respectively). The following antibodies were used: anti-annexin 1 (Santa Cruz, Dallas, TX, USA; Sc-11387, 1 : 1000), anti-histone H3 (Cell Signaling Technology, Beverly, MA, USA; no. 4499, 1: 1000), anti-p53 (Santa Cruz; sc-126, 1: 1000), anti-cleaved caspase-3 (Cell Signaling Technology; no. 9664, 1:1000), anti-cleaved PARP (Cell Signaling Technology; no. 5625, 1 : 1000), anti-GFP (Santa Cruz; sc-9996, $1: 1000$ ), anti-His (Santa Cruz; sc-803, 1 : 1000), anti- $\alpha$-tubulin (Santa Cruz; sc-53646, $1: 1000$ ), and anti- $\beta$-actin (Santa Cruz; sc-47778, $1: 1000$ ).

Cell culture. Primary cultured rat cerebral cortical neurons were prepared from 16- to 18- day-old Sprague-Dawley rat embryos, as described previously. ${ }^{38}$ The protocol for the use of rats for neuronal cultures was performed according to the principles of the Animal Care Committee of Huazhong University of Science and Technology. Briefly, rat embryos were decapitated and the tissues of the cerebral cortex were isolated under a dissection microscope, cut into $\sim 1-\mathrm{mm}^{3}$ pieces, and incubated with $0.25 \%$ trypsin-EDTA for $15 \mathrm{~min}$ at $37^{\circ} \mathrm{C}$. Freshly prepared Dulbecco's modified Eagle's medium: nutrient mixture F12 (DMEM-F12) medium containing 10\% fetal bovine serum (FBS; Gibco, Gaithersburg, MD, USA) was used to stop the trypsin digestion. Subsequently, fire-polished glass pipettes were used to gently triturate the tissue mass into a cell suspension. Finally, the cells were counted and plated in poly-L-lysine-coated culture dishes or coverslips in 24-well plates at a density of $1 \times 10^{6}$ cells per dish or $2 \times 10^{5}$ cells per well, respectively. Neurons were maintained at $37{ }^{\circ} \mathrm{C}$ in a humidified atmosphere containing $5 \% \mathrm{CO}_{2}$. After $24 \mathrm{~h}$, the culture medium was replaced with Neurobasal Medium supplemented with $2 \%$ B-27, and the cultures were fed two times per week. Neurons were used for the experiments between days 7 and 10 in vitro.

HEK293 cells were maintained in DMEM supplemented with 10\% FBS (Gibco), penicillin $(100 \mathrm{U} / \mathrm{ml}) /$ streptomycin $(100 \mu \mathrm{g} / \mathrm{ml})$ and $2 \mathrm{mM} \mathrm{L}$-glutamine at $37^{\circ} \mathrm{C}$ in a $5 \% \mathrm{CO}_{2}$. Confluent cell layers were split two times per week. Transfections were performed using Lipofectamine 2000 (Invitrogen) when the cells were $80-90 \%$ confluent.

Plasmids. DNA fragments corresponding to the full-length ANXA1 and p53 coding sequences were amplified by PCR, followed by cloning into the pcDNA3.0 plasmid or pEGFP-N1 vector (Invitrogen). To express ANXA1 tagged with red 

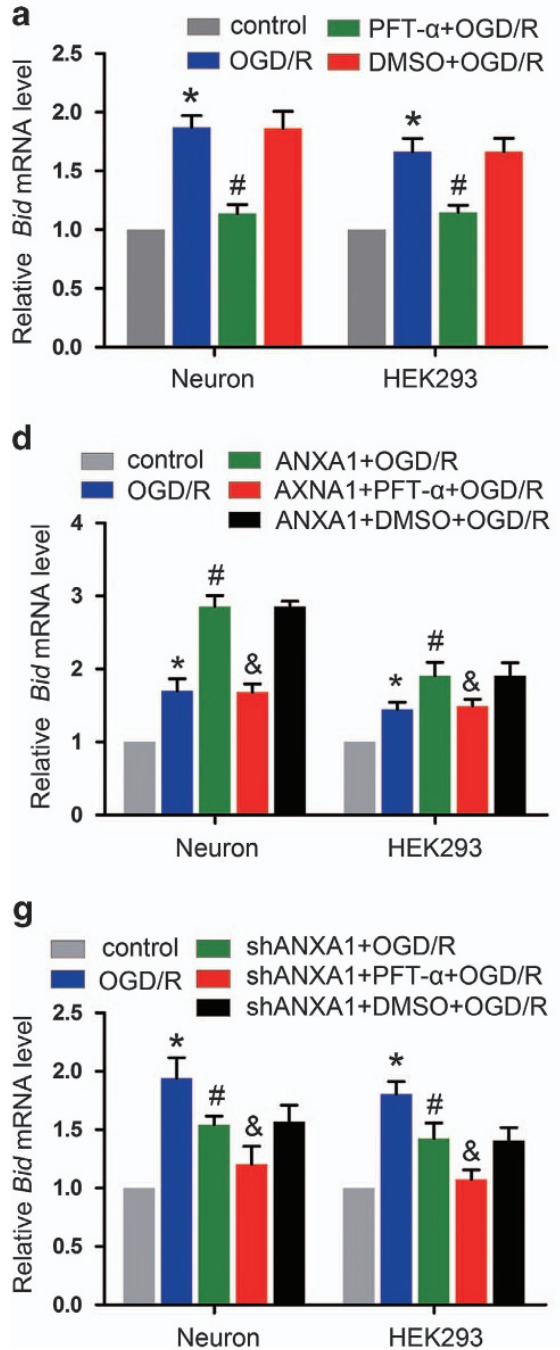
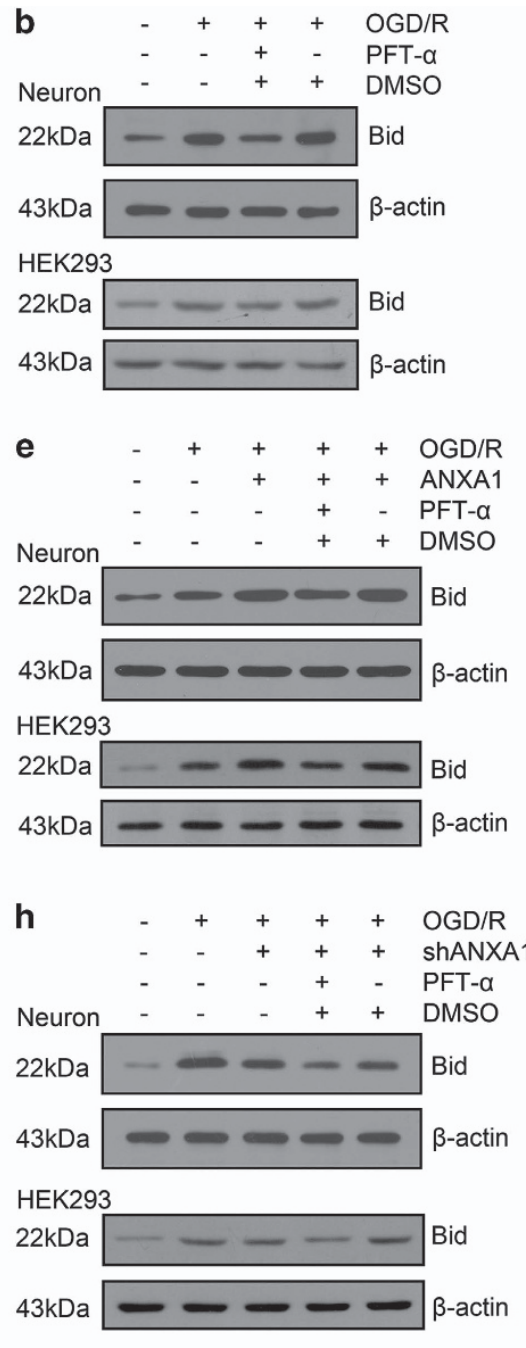

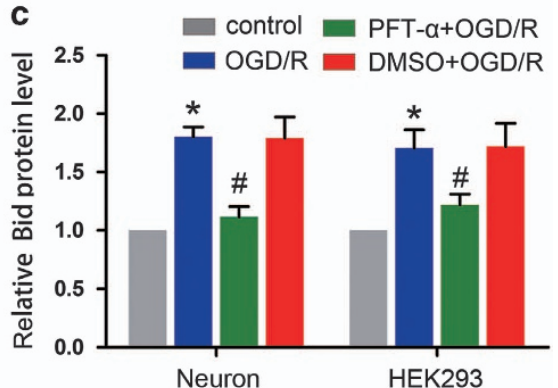

f

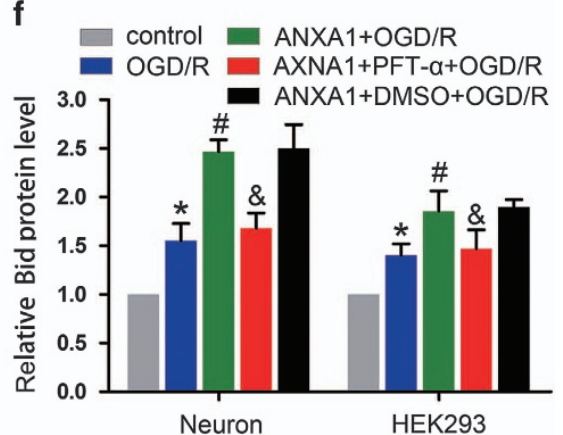

i

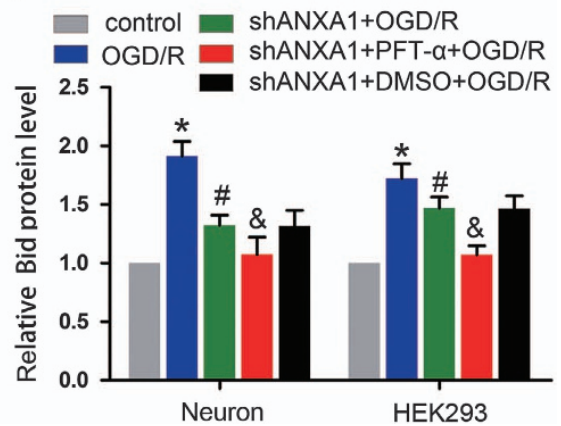

Figure 6 ANXA1 coregulates the expression of Bid with p53. (a) qPCR results showing the expression of Bid mRNA after treatment with PFT- $\alpha$. The data are expressed as the means \pm S.E.M. from three independent experiments. ${ }^{*} P<0.05$ versus control and ${ }^{\sharp} P<0.05$ versus $O G D / R$. (b) Western blot showing expression of the Bid protein after treatment with PFT- $\alpha$ in primary cultured neurons or HEK293 cells. (c) Statistical analysis of the data shown in (b). The data are expressed as the means \pm S.E.M. from three independent experiments. ${ }^{*} P<0.05$ versus control and ${ }^{\#} P<0.05$ versus $\mathrm{OGD} / \mathrm{R}$. (d) Statistical analysis of qPCR results showing the expression of Bid mRNA after treatment with ANXA1 and PFT- $\alpha$. The data are expressed as the means \pm S.E.M. from three independent experiments. ${ }^{*} P<0.05$ versus control, ${ }^{\#} P<0.05$ versus 0 GD/R and ${ }^{\circledR} P<0.05$ versus ANXA1+OGD/R. (e) Western blot showing expression of the Bid protein after treatment with ANXA1 and PFT- $\alpha$ in primary cultured neurons or HEK293 cells. (f) Statistical analysis of the data shown in (e). The data are expressed as the means \pm S.E.M. from three independent experiments. ${ }^{*} P<0.05$ versus control, ${ }^{\#} P<0.05$ versus 0 GD/R and ${ }^{\&} P<0.05$ versus ANXA1+OGD/R. (g) Statistical analysis of the qPCR results shows the expression of Bid mRNA after treatment with ANXA1 shRNA and PFT- $\alpha$. The data are expressed as the means \pm S.E.M. from three independent experiments. ${ }^{*} P<0.05$ versus control, ${ }^{\#} P<0.05$ versus 0 GD/R and ${ }^{\&} P<0.05$ versus ANXA1 shRNA+OGD/R. (h) Western blot showing expression of the Bid protein after treatment with ANXA1 shRNA and PFT- $\alpha$. (i) Statistical analysis of the data shown in (h). The data are expressed as the means \pm S.E.M. from three independent experiments. ${ }^{*} P<0.05$ versus control, ${ }^{\#} P<0.05$ versus 0 GD/R and ${ }^{\circledR} P<0.05$ versus $A N X A 1$ shRNA+OGD/R

fluorescence protein, the pjRED-C1-ANXA1 plasmid was constructed by PCR amplification of the ANXA1 coding sequence, followed by cloning into the pjRed-C1 vector (gift from Professor He Li, HUST, China). Human ANXA1 shRNA plasmids were purchased from GenePharma (Suzhou, Wuhan, China). The target sequence for ANXA1 (GenBank No. NM_000700.2) shRNA no. 1 was 5'-AGCTTGAG ACCATCAAGGG-3', and the target sequence for ANXA1 shRNA no. 2 was 5'-AGAACAACTTGTATAGGGT-3'.

Adenoviral infection. The adenoviral vectors carried GFP, rat ANXA1 and ANXA1 shRNA or the Scr were constructed by Vigene Biosciences Co. Ltd. (Shandong, China). The shRNA sequence that targets rat ANXA1 sequence (GenBank No. NM_012904.2) was designed as follows: 5'-GCCTCACAACCATTGTGAAGT- $3^{\prime}$, and a Scr shRNA served as a negative control. Primary cultured neurons were infected with ANXA1, ANXA1 shRNA, or the Scr adenoviral particles. The optimal multiplicity of infection was determined to be $50: 1$ to $100: 1$, based on the observed fluorescence intensity of GFP. After the adenovirus infection for $48 \mathrm{~h}$, cells were subjected to OGD/R and (or) other treatments.

Establishment of the OGD/R model. The cell culture medium was replaced with glucose-free DMEM equilibrated with nitrogen, after which neuron and HEK293 cells were transferred to an incubator containing $5 \% \mathrm{CO}_{2}$ and $95 \% \mathrm{~N}_{2}$ at $37^{\circ} \mathrm{C}$ for $1 \mathrm{~h}$. After washing the cultures with DMEM three times, the cultures were maintained in glucose-containing normoxic DMEM at $37^{\circ} \mathrm{C}$ in a humidified $5 \% \mathrm{CO}_{2}$ incubator. In all experiments, the culture medium $\mathrm{pH}$ was maintained at 7.2 . 
Protein extraction and preparation. Subcellular fractionation was performed as described previously. ${ }^{9}$ Cell pellets were resuspended with buffer $\mathrm{A}$ (40 mM Tris- $\mathrm{HCl}, 10 \mathrm{mM} \mathrm{NaCl}, 1 \mathrm{mM}$ EDTA, $1 \mathrm{mM}$ DTT, and protease inhibitors). Resuspended cells were incubated on ice for $15 \mathrm{~min}$ and mixed by vortexing every $5 \mathrm{~min}$ for $5 \mathrm{~s}$. Then, $30 \mu \mathrm{l} 10 \% \mathrm{NP}-40$ was added to the cell extracts and vigorously shaken for $10 \mathrm{~s}$. After centrifugation at $14000 \times \mathrm{g}$ for $10 \mathrm{~min}$ at $4{ }^{\circ} \mathrm{C}$, the supernatants were transferred to a new tube (the cytosolic fraction). The pellets were resuspended with buffer B $(40 \mathrm{mM}$ Tris- $\mathrm{HCl}, 420 \mathrm{mM} \mathrm{NaCl}, 10 \%$ glycerol, $1 \mathrm{mM}$ EDTA, $1 \mathrm{mM}$ DTT, and protease inhibitors). The resuspended extracts were incubated on ice for $20 \mathrm{~min}$ and mixed vigorously by vortexing every $5 \mathrm{~min}$ for $5 \mathrm{~s}$. After centrifugation at $14000 \times \mathrm{g}$ for $10 \mathrm{~min}$ at $4{ }^{\circ} \mathrm{C}$, the supernatants were transferred to a new tube (the nuclear fraction).

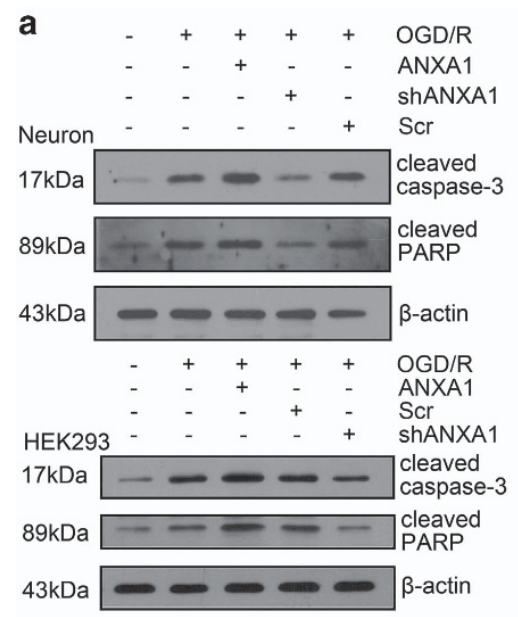

e
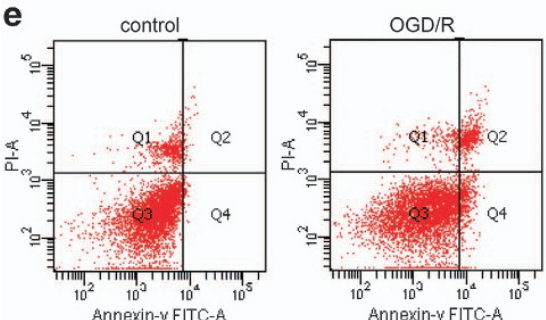

ANXA1+OGD/R+BI-6C9
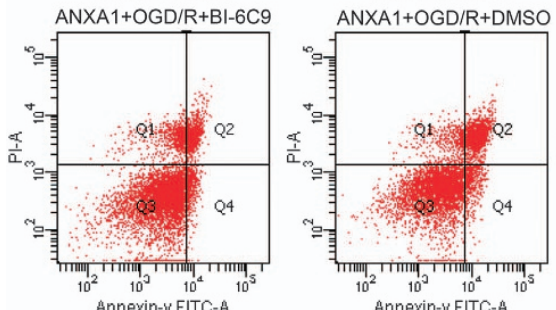

Annexin-y FITC-A b

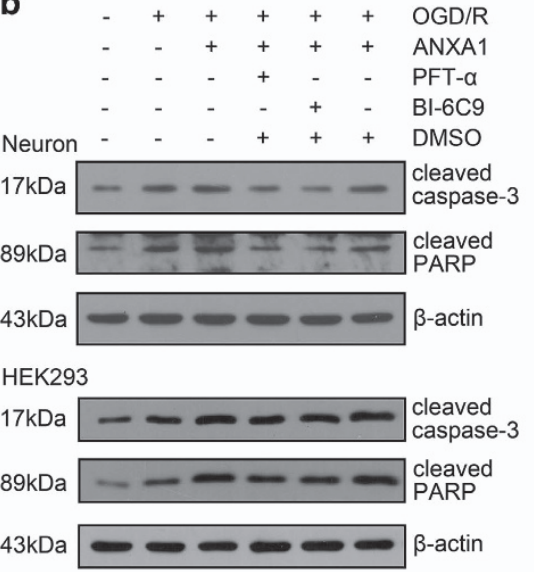

c
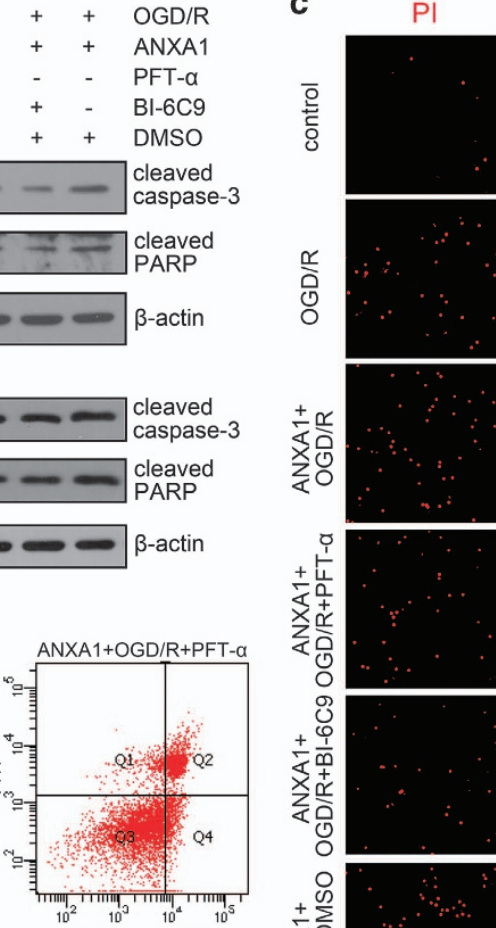

Annexin-Y FITC-A

$\mathrm{SCr}+\mathrm{OGD} / \mathrm{R}$

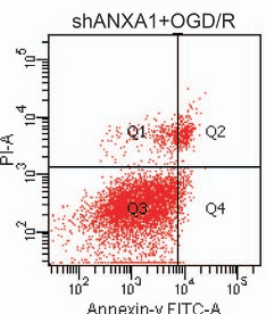

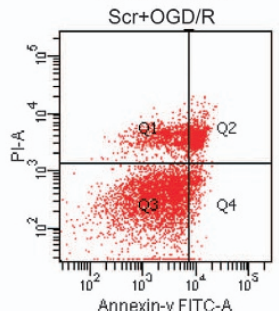

Annexin-y FITC-A

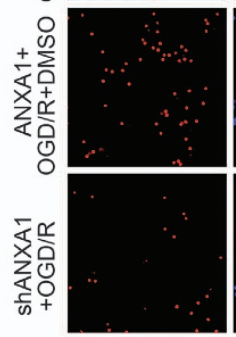

d

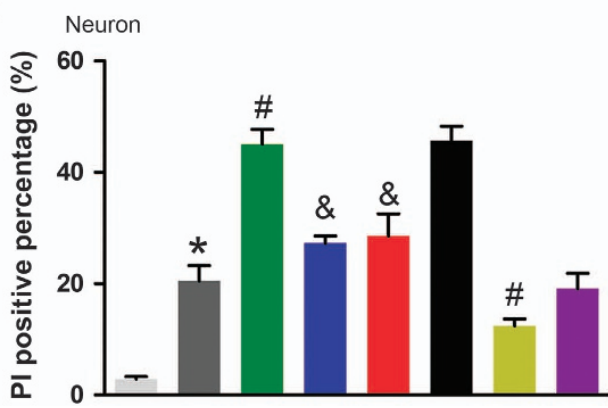

f HEK293

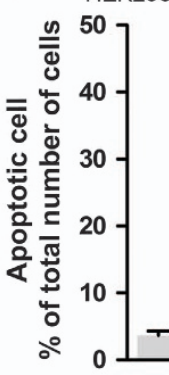

DAPI
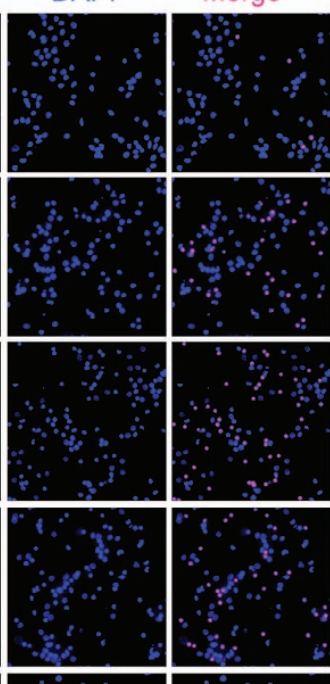
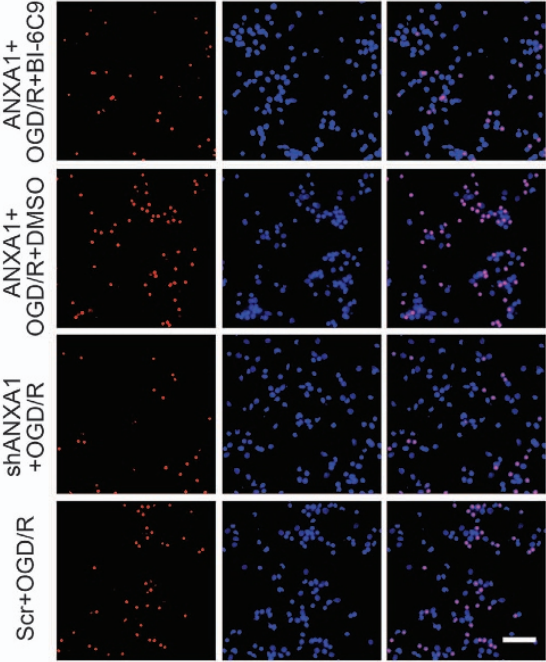

Figure 7 Effect of ANXA1 on the caspase-3 apoptotic pathway. (a) Western blot showing the expression of cleaved caspase-3 and PARP following treatment with ANXA1 or ANXA1 shRNA in neuronal cells and HEK293 cells. The results were obtained in three independent experiments. (b) Western blot showing the expression of cleaved caspase-3 and PARP following treatment with ANXA1, PFT- $\alpha$, or BI-6C9 in primary cultured neurons or HEK293 cells. The results were obtained in three independent experiments. (c) PI staining results demonstrating cell death following treatment with ANXA1, PFT- $\alpha, \mathrm{BI}-6 \mathrm{C} 9$, or ANXA1 shRNA in primary cultured neurons. Scale bar $=50 \mu \mathrm{m}$. (d) Statistical analysis of the data shown in (c). The data are expressed as the means \pm S.E.M. from three independent experiments. ${ }^{*} P<0.05$ versus control, ${ }^{\#} P<0.05$ versus 0 GD/R and ${ }^{\&} P<0.05$ versus ANXA1+OGD/R. (e) Flow cytometry results demonstrating apoptosis following treatment with ANXA1, PFT- $\alpha, \mathrm{BI}-6 \mathrm{C} 9$, , or ANXA1 shRNA in HEK293 cells. (f) Statistical analysis of the data shown in (e). The data are expressed as the means \pm S.E.M. from three independent experiments. ${ }^{*} P<0.05$ versus control, ${ }^{\#} P<0.05$ versus $\mathrm{OGD} / \mathrm{R}$ and ${ }^{\&} P<0.05$ versus ANXA1+OGD/R 


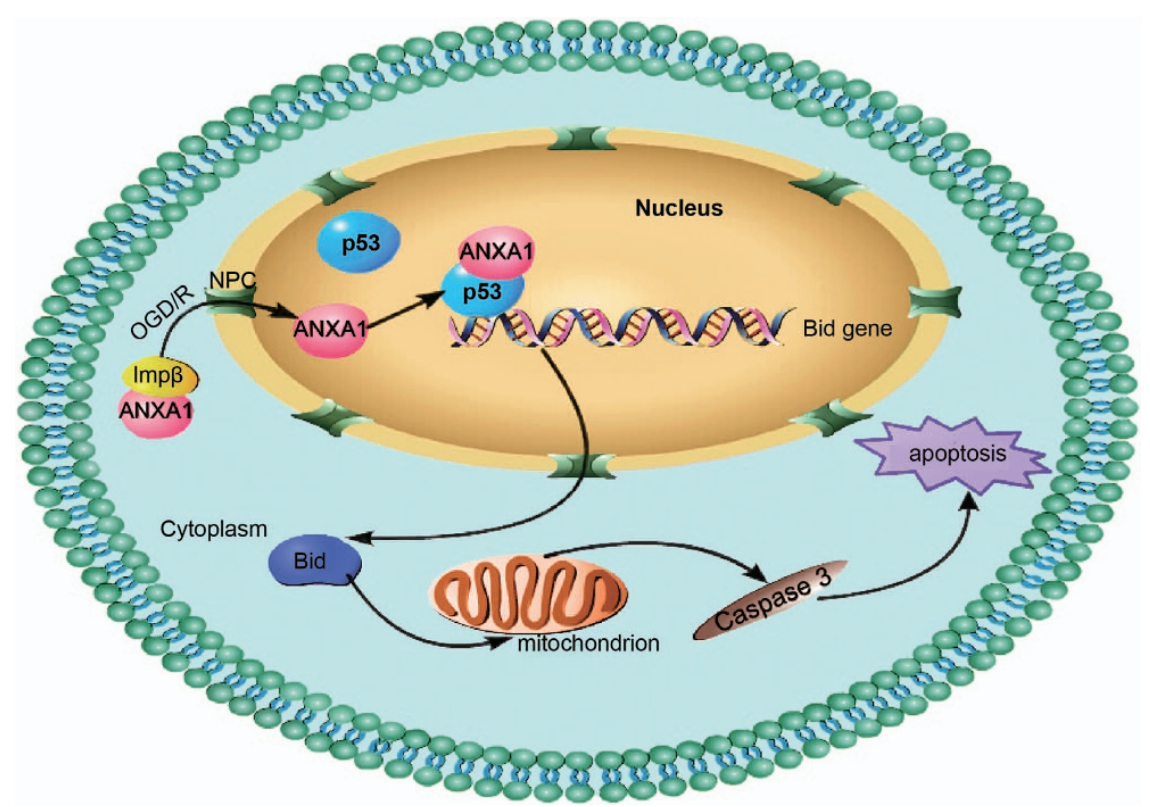

Figure 8 Schematic representation of the contribution of ANXA1 to apoptosis after OGD/R. Nuclear ANXA1 translocation is mediated by $\operatorname{Imp} \beta$ function after OGD/R and induces apoptosis via the p53-Bid-caspase-3 pathway

Western blot analysis. Proteins were run on 10 or $12 \%$ polyacrylamide gels and transferred to polyvinylidene difluoride (PVDF) membranes. PVDF membranes were blocked with $5 \%$ bovine serum albumin at room temperature for $60-90 \mathrm{~min}$ and incubated overnight at $4{ }^{\circ} \mathrm{C}$ with antigen-specific primary antibodies. Blots were then incubated with species-specific HRP-conjugated secondary antibodies for $60 \mathrm{~min}$ at room temperature. Proteins were visualized by incubation with a Chemiluminescence Substrate Kit (ECL Plus; Perkin-Elmer Inc., Covina, CA, USA). The expression of the target proteins was quantified using the ImageJ software ( $\mathrm{NIH}$, Bethesda, MD, USA) after normalizing to $\beta$-actin, $\alpha$-tubulin, or histone $\mathrm{H} 3$ expression.

Co-IP. CO-IP was performed as described previously. ${ }^{7}$ Briefly, cell lysates were generated by sonication in a buffer containing $20 \mathrm{mM}$ HEPES, $400 \mathrm{mM} \mathrm{KCl}, 5 \%$ glycerol, $5 \mathrm{mM}$ EDTA, $0.4 \% \mathrm{NP}-40$, and protease inhibitors, and precleared by centrifugation. The cell lysates were then incubated with an anti-GFP or anti-p53 antibody overnight at $4{ }^{\circ} \mathrm{C}$. The reaction mixture was then incubated with protein $\mathrm{A} /$ $\mathrm{G}$ PLUS-Agarose beads (Santa Cruz; sc-2003) for $2 \mathrm{~h}$ at $4{ }^{\circ} \mathrm{C}$. The precipitates were washed three times with wash buffer and then eluted from the protein $A / G$ PLUS-Agarose beads by boiling with $1 \times$ sodium dodecyl sulfate (SDS) for 5 min at $95^{\circ} \mathrm{C}$. The protein samples were resolved by SDS-polyacrylamide gel electrophoresis.

Immunofluorescence. Indirect immunofluorescence analysis was performed as described previously. ${ }^{9}$ The cultured neurons (fixed in $4 \%$ paraformaldehyde) were gently washed two times with PBS preheated to $37^{\circ} \mathrm{C}$. The cells were then subjected to treatment with $10 \%$ Triton X-100 for $10 \mathrm{~min}$ to rupture the cell membranes. The fixed cells were again washed with PBS for $15 \mathrm{~min}$, and the cells were again treated with $5 \%$ bovine serum albumin for another $40 \mathrm{~min}$ to block nonspecific binding. The cells were then incubated with a rabbit polyclonal ANXA1 antibody $(1: 200)$ or a mouse monoclonal p53 antibody $(1: 200)$ in $5 \%$ bovine serum albumin at $4{ }^{\circ} \mathrm{C}$ overnight. The cells were washed and subsequently incubated with secondary antibody in $5 \%$ bovine serum albumin at $37^{\circ} \mathrm{C}$ for $1 \mathrm{~h}$. A Zeiss double-photon fluorescence microscope (Zeiss 510 Meta, Oberkochen, Germany) was used to detect the fluorescence.

Quantitative real-time PCR. Cells were transfected with or without ANXA1, ANXA1 shRNA or Scr. After transfection, total RNA was prepared with the TRIzol reagent (Invitrogen), and cDNA was synthesized from $1 \mu \mathrm{g}$ RNA using the ReverTra Ace- $\alpha$-TM First Strand cDNA Synthesis Kit (Toyobo, Osaka, Japan). qPCR was performed with SYBR Green Real-Time PCR Master Mix (Toyobo) on a C1000 Thermal Cycler (Bio-Rad Laboratories, Hercules, CA, USA), according to the manufacturer's recommendations. The primers used were as follows: rat Bid, 5'-CGACGAGGTGAAGACATCCT-3' (forward primer) and 5'-AGCAGAGATGGTGCATGACT-3' (reverse primer); human Bid, 5'-ACTGGTGTTTGGCTTCCTCC-3' (forward primer) and 5'-ATTCTTCCCAAGCGGGAGTG-3' (reverse primer); rat $\beta$-actin, 5'-TAAGGCCAACCGTGAAAAGAT-3' (forward primer) and $5^{\prime}$-GGTACGACCAGAGGCATACA-3' (reverse primer). Human $\beta$-actin, $5^{\prime}$-TCCACCACCCTGTTGCTGTA-3' (forward primer) and 5'-ACCACAGTCCATGCCATCAC-3' (reverse primer).

Luciferase reporter assay. Cells were routinely co-transfected with a TKRenilla luciferase plasmid (Promega, Madison, WI, USA) to normalize for the transfection efficacy. To determine luciferase expression, the pGL4.38 [luc2P/p53 RE/Hygro] Vector (Promega) was transfected into HEK293 cells 1 day after plating. The indicated treatments were performed $24 \mathrm{~h}$ after transfection, and the cells were collected according to the manufacturer's instructions (Roche Diagnostics, Roswell, GA, USA). Briefly, the cell lysates were prepared with $1 \times$ lysis buffer, and the luciferase reporter signals were detected immediately after adding luciferin substrates using a Fluoroskan Ascent FL System (Thermo Scientific, Waltham, MA, USA). The data shown represent the mean values obtained from three independent experiments.

Flow cytometry. The effects of ANXA1 on apoptosis were evaluated using a FITC-Annexin V Apoptosis Detection Kit (BD Pharmingen, San Diego, CA, USA). Briefly, $10^{5}$ cells were harvested through trypsinization and washed two times with cold PBS. The cells were then centrifuged at 1000 r.p.m. for $5 \mathrm{~min}$, and the supernatant was discarded and the pellet was resuspended in $500 \mu \mathrm{l} 1 \times$ Annexin V-binding buffer in a 1.5-ml culture tube and later incubated with $5 \mu \mathrm{l}$ of an FITCconjugated Annexin $\mathrm{V}$ and $5 \mu \mathrm{lPI}$ for $10 \mathrm{~min}$ at room temperature in the dark. The samples were analyzed by fluorescence-activated cell sorting using the Cell Quest Research software (Beckman Coulter, Brea, CA, USA).

Statistical analysis. Data are expressed as the mean value \pm S.E.M. Statistical significance was calculated using the Student's $t$-test. A value of $P<0.05$ was considered statistically significant. All results shown are representative of at least three independent experiments. 


\section{Conflict of Interest}

The authors declare no conflict of interest.

Acknowledgements. The current studies were supported by funding from the National Natural Science Foundation of China (program number 31171029).

1. Eltzschig HK, Eckle T. Ischemia and reperfusion - from mechanism to translation. Nat Med 2011; 17: 1391-1401.

2. Culmsee $\mathrm{C}$, Krieglstein J. Ischaemic brain damage after stroke: new insights into efficient therapeutic strategies. International Symposium on Neurodegeneration and Neuroprotection. EMBO Rep 2007; 8: 129-133.

3. Duehrkop C, Rieben R. Ischemia/reperfusion injury: effect of simultaneous inhibition of plasma cascade systems versus specific complement inhibition. Biochem Pharmacol 2014; 88: 12-22.

4. Lo EH, Dalkara T, Moskowitz MA. Mechanisms, challenges and opportunities in stroke. Nat Rev Neurosci 2003; 4: 399-415.

5. Moskowitz MA, Lo EH, ladecola C. The science of stroke: mechanisms in search of treatments. Neuron 2010; 67: 181-198.

6. Andrabi SA, Kang HC, Haince JF, Lee YI, Zhang J, Chi Z et al. Iduna protects the brain from glutamate excitotoxicity and stroke by interfering with poly(ADP-ribose) polymer-induced cell death. Nat Med 2011; 17: 692-699.

7. Tu W, Xu X, Peng L, Zhong X, Zhang W, Soundarapandian MM et al. DAPK1 interaction with NMDA receptor NR2B subunits mediates brain damage in stroke. Cell 2010; 140: 222-234.

8. Culmsee C, Zhu C, Landshamer S, Becattini B, Wagner E, Pellecchia M et al. Apoptosis-inducing factor triggered by poly(ADP-ribose) polymerase and Bid mediates neuronal cell death after oxygen-glucose deprivation and focal cerebral ischemia. J Neurosci 2005; 25: 10262-10272.

9. Zhao Y, Wang J, Jiang H, Yu Z, Li X, Shi J. Following OGD/R, annexin 1 nuclear translocation and subsequent induction of apoptosis in neurons are assisted by myosin IIA in a TRPM7 kinase-dependent manner. Mol Neurobiol 2015; 51: 729-742.

10. Lim LH, Pervaiz S. Annexin 1: the new face of an old molecule. FASEB J 2007; 21: 968-975.

11. Solito E, McArthur S, Christian H, Gavins F, Buckingham JC, Gillies GE. Annexin A1 in the brain - undiscovered roles? Trends Pharmacol Sci 2008; 29: 135-142.

12. Kim YS, Ko J, Kim IS, Jang SW, Sung HJ, Lee HJ et al. PKC-dependent cleavage and nuclear translocation of annexin A1 by phorbol 12-myristate 13-acetate. Eur $\mathrm{J}$ Biochem 2003; 270: 4089-4094.

13. Wang ZM, Zhu SG, Wu ZW, Lu Y, Fu HZ, Qian RQ. Kirenol upregulates nuclear annexin-1 which interacts with NF-кB to attenuate synovial inflammation of collagen-induced arthritis in rats. J Ethnopharmacol 2011; 137: 774-782.

14. Culmsee C, Plesnila N. Targeting Bid to prevent programmed cell death in neurons. Biochem Soc Trans 2006; 34: 1334-1340.

15. Engel T, Plesnila N, Prehn JH, Henshall DC. In vivo contributions of BH3-only proteins to neuronal death following seizures, ischemia, and traumatic brain injury. J Cereb Blood Flow Metab 2011; 31: 1196-1210.

16. Sax JK, Fei P, Murphy ME, Bernhard E, Korsmeyer SJ, El-Deiry WS. BID regulation by p53 contributes to chemosensitivity. Nat Cell Biol 2002; 4: 842-849.

17. Maas C, de Vries E, Tait SW, Borst J. Bid can mediate a pro-apoptotic response to etoposide and ionizing radiation without cleavage in its unstructured loop and in the absence of p53. Oncogene 2011; 30: 3636-3647.

18. Beckerman R, Prives C. Transcriptional regulation by p53. Cold Spring Harb Perspect Biol 2010; 2: a000935.

19. Manfredi JJ. P53 and apoptosis: it's not just in the nucleus anymore. Mol Cell 2003; 11: $552-554$

20. Slee EA, Keogh SA, Martin SJ. Cleavage of BID during cytotoxic drug and UV radiationinduced apoptosis occurs downstream of the point of Bcl-2 action and is catalysed by caspase-3: a potential feedback loop for amplification of apoptosis-associated mitochondrial cytochrome c release. Cell Death Differ 2000; 7: 556-565.
21. Landshamer S, Hoehn M, Barth N, Duvezin-Caubet S, Schwake G, Tobaben S et al. Bid-induced release of AIF from mitochondria causes immediate neuronal cell death. Cell Death Differ 2008; 15: 1553-1563.

22. Kook S, Zhan X, Cleghorn WM, Benovic JL, Gurevich VV, Gurevich EV. Caspase-cleaved arrestin-2 and BID cooperatively facilitate cytochrome $C$ release and cell death. Cell Death Differ 2014; 21: 172-184.

23. Heinrich M, Neumeyer J, Jakob M, Hallas C, Tchikov V, Winoto-Morbach S et al. Cathepsin D links TNF-induced acid sphingomyelinase to Bid-mediated caspase-9 and-3 activation. Cell Death Differ 2004; 11: 550-563.

24. Yin XM. Bid, a BH3-only multi-functional molecule, is at the cross road of life and death. Gene 2006; 369: 7-19.

25. Kastan MB, Berkovich E. P53: a two-faced cancer gene. Nat Cell Biol 2007; 9: 489-491.

26. Freed-Pastor WA, Prives C. Mutant p53: one name, many proteins. Genes Dev 2012; 26 : 1268-1286.

27. Aylon Y, Oren M. Living with p53, dying of p53. Cell 2007; 130: 597-600.

28. Wang DB, Kinoshita C, Kinoshita Y, Morrison RS. P53 and mitochondrial function in neurons. Biochim Biophys Acta 2014; 1842: 1186-1197.

29. Soderholm JF, Bird SL, Kalab P, Sampathkumar Y, Hasegawa K, Uehara-Bingen M et al. Importazole, a small molecule inhibitor of the transport receptor importin-beta. Acs Chem Biol 2011; 6: 700-708.

30. Wagstaff KM, Sivakumaran $\mathrm{H}$, Heaton SM, Harrich D, Jans DA. Ivermectin is a specific inhibitor of importin alpha/beta-mediated nuclear import able to inhibit replication of HIV-1 and dengue virus. Biochem J 2012; 443: 851-856.

31. Dorovkov MV, Ryazanov AG. Phosphorylation of annexin I by TRPM7 channel-kinase. $J$ Biol Chem 2004; 279: 50643-50646.

32. D'Acunto CW, Gbelcova H, Festa M, Ruml T. The complex understanding of Annexin A1 phosphorylation. Cell Signal 2014; 26: 173-178.

33. Rosengarth A, Gerke V, Luecke H. X-ray structure of full-length annexin 1 and implications for membrane aggregation. J Mol Biol 2001; 306: 489-498.

34. Park JJ, Lim KH, Baek KH. Annexin-1 regulated by HAUSP is essential for UV-induced damage response. Cell Death Dis 2015; 6: e1654.

35. Stuqui B, de Paula-Silva M, Carlos CP, Ullah A, Arni RK, Gil CD et al. Ac2-26 mimetic peptide of annexin A1 inhibits local and systemic inflammatory processes induced by Bothrops moojeni venom and the Lys-49 phospholipase A2 in a rat model. PLoS One 2015; 10: e0130803.

36. Perretti M, Gavins FN. Annexin 1: an endogenous anti-inflammatory protein. News Physiol Sci 2003; 18: 60-64.

37. Girol AP, Mimura KK, Drewes CC, Bolonheis SM, Solito E, Farsky SH et al. Anti-inflammatory mechanisms of the annexin $A 1$ protein and its mimetic peptide Ac2-26 in models of ocular inflammation in vivo and in vitro. $J$ Immunol 2013; 190 : 5689-5701.

38. Zhang J, Zhao F, Zhao Y, Wang J, Pei L, Sun $\mathrm{N}$ et al. Hypoxia induces an increase in intracellular magnesium via transient receptor potential melastatin 7 (TRPM7) channels in rat hippocampal neurons in vitro. J Biol Chem 2011; 286: 20194-20207.

Cell Death and Disease is an open-access journal published by Nature Publishing Group. This work is licensed under a Creative Commons Attribution 4.0 International License. The images or other third party material in this article are included in the article's Creative Commons license, unless indicated otherwise in the credit line; if the material is not included under the Creative Commons license, users will need to obtain permission from the license holder to reproduce the material. To view a copy of this license, visit http://creativecommons.org/licenses/by/4.0/

C) The Author(s) 2016

Supplementary Information accompanies this paper on Cell Death and Disease website (http://www.nature.com/cddis) 